\title{
CARACTERIZAÇÃO DA COMUNICAÇÃO ENTRE ENFERMEIROS E PACIENTES DE UM HOSPITAL DO CEARÁ, BRASIL
}

Patrícia de Lemos Negreiros ${ }^{1}$, Mayara de Oliveira Fernandes ${ }^{2}$, Kátia Nêyla de Freitas Macêdo-Costa ${ }^{3}$, Grazielle Roberta Freitas da Silva ${ }^{4}$.

'Enfermeira, Ceará

${ }^{2}$ Enfermeira. Universidade Estadual Vale do Acaraú, Ceará.

${ }^{3}$ Doutora em Enfermagem. Docente da Universidade Federal da Paraíba. ${ }^{4}$ Doutora em Enfermagem. Docente da Universidade Federal do Piauí campus, Piauí, Brasil

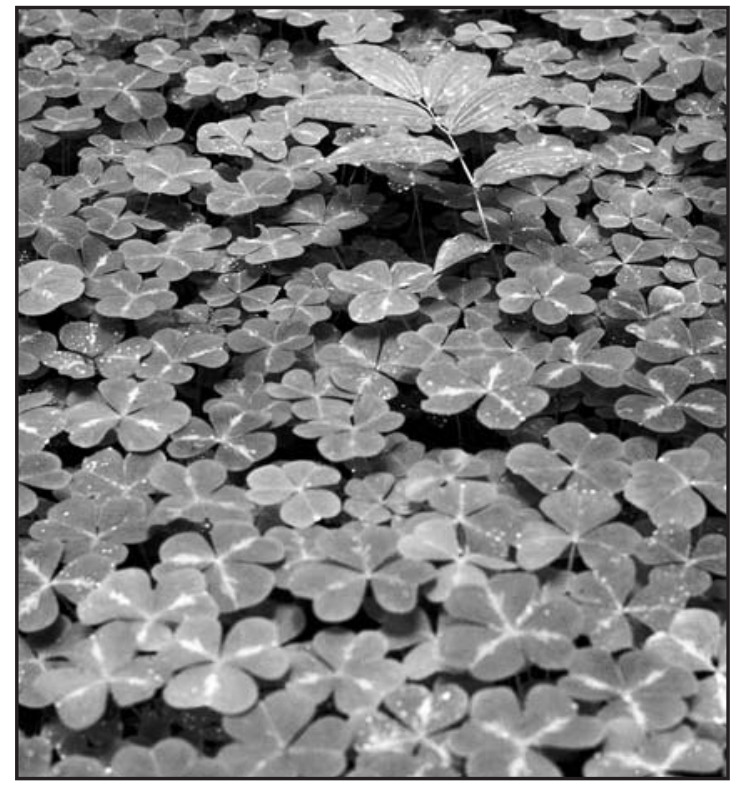

CHARACTERIZATION OF THE THERAPEUTIC COMMUNICATION OF NURSES IN A HOSPITAL OF CEARÁ, BRAZIL

\section{ABSTRACT}

Introduction: The communication between nurses and patients in the hospital environment excessively intervenes with the process of treatment of the health. From the moment that imperfections, these can be identified to improve the interaction and the assistance of nursing.

Aims: To characterize the therapeutical communication between nurses and patients in the hos- pital environment according to estimated of Stefanelli.

Material and Method: Study description with carried through quantitative boarding in a hospital of the state of the Ceará in julho/2007. The citizens of the study had been five nurses. Comment of 21 interactions established between nurses and patients was carried through. For the registers of the data a suitable instrument's Stefanelli was used daily of field.

Results: The not-therapeutical's item had been gifts in the interactions of the majority of the nurses, occurring 32 times during the 21 interactions. Intent it the fact of that, the used item more was the use of professional jargon. The unidirectional communication occurred frequently.

Conclusions: The nurses if communicate little of efficient and not directed form during the interactions that establish with the patients of the hospital.

Key words: Nursing; Communication; Hospital.

CARACTERIZACIÓN DE LA COMUNICACIÓN TERAPÉUTICA DE ENFERMERAS EN UN HOSPITAL DE CEARÁ, BRASIL

\section{RESUMEN}

Introducción: La comunicación entre las enfermeras y los pacientes en el ambiente del hospital interviene excesivamente con el proceso del tratamiento de la salud. A partir del momento que las imperfecciones, éstas se pueden identificar para 
mejorar la interacción y la ayuda del rol de la enfermera.

Objetivo: caracterizar la comunicación terapéutica entre las enfermeras y los pacientes en el ambiente del hospital según Stefanelli.

Material y método: Estudio descriptivo cuantitativo en un hospital del Ceará, Brasil en junio/2007. Los ciudadanos del estudio habían sido cinco enfermeras. El comentario de 21 interacciones establecidas entre las enfermeras y los pacientes fue ejecutado. Para los registros de los datos uso diario del campo y instrumento adaptado del Stefanelli.

Resultados: El artículo no-terapéutico había estado presente en las interacciones de la mayoría de las enfermeras, ocurriendo 32 veces durante las 21 interacciones. Intento el hecho de eso, el artículo usado era más el uso de la jerga profesional. La comunicación unidireccional ocurrió con frecuencia.

Conclusiones: Las enfermeras se comunican de forma poco eficiente y no dirigida durante las interacciones que establecen con los pacientes del hospital.

Palabras clave: Enfermería; Comunicación, Hospital.

\section{RESUMO}

Introdução: A comunicação entre enfermeiros e pacientes no ambiente hospitalar interfere sobremaneira no processo de tratamento e reestabelecimento da saúde. A partir do momento que existem falhas, essas possam ser identificadas para aprimorar a interação e a assistência de enfermagem.

Objetivo: Caracterizar a comunicação terapêutica entre enfermeiros e pacientes no ambiente hospitalar segundo os pressupostos de Stefanelli (1993).

Materiais e Métodos: Estudo descritivoexploratório, com abordagem quantitativa realizado em um hospital do estado do Ceará em julho/2007. Os sujeitos do estudo foram cinco enfermeiros. Foi realizada observação de 21 interações estabelecidas entre enfermeiros e pacientes. Para os registros dos dados utilizou-se um diário de campo e o instrumento adaptado de Stefanelli.
Resultados: Os itens não-terapêuticos estiveram presentes nas interações da maioria dos enfermeiros, ocorrendo 32 vezes durante as 21 interações. Atenta-se ao fato de que, o item mais utilizado foi o uso de jargão profissional. A comunicação unidirecional ocorreu com freqüência.

Considerações finais: Os enfermeiros se comunicam de forma pouco eficaz e não direcionada durante as interações que estabelecem com os pacientes da instituição.

Descritores: Enfermagem; Comunicação; Hospital.

\section{INTRODUÇÃO}

O profissional de enfermagem possui contato direto com o paciente durante a hospitalização. Nesse sentido, pode-se enfatizar a importância do enfermeiro em codificar, decifrar e perceber o significado da mensagem que o paciente envia. O profissional poderá estabelecer um plano de cuidados adequado, eficaz e coerente às necessidades dos pacientes (Chubaci, Merighi, 2002). O processo comunicativo no ambiente hospitalar pode ser definido como toda interação na qual o enfermeiro esteja voltado para atender as reais necessidades do paciente que precisa de ajuda específica nesse ambiente (Paula, Furegato, Scatena, 2000).

Uma questão que merece destaque são as possíveis falhas no processo comunicativo entre enfermeiros e pacientes e a pouca importância que parece ser dado à comunicação durante a formação acadêmica.

Estudo de Sadala e Stefanelli (1996) mostra barreiras comunicacionais encontradas por alunos e profissionais recém-formados na prática assistencial hospitalar são algumas: a falta de domínio do processo de comunicação, o não estabelecimento de limites para o paciente, a ansiedade e as dificuldades para o oferecimento de apoio. Observa-se diante das falhas supracitadas, que as barreiras existentes durante o cuidar, remetem a um não conhecimento ou mesmo, a uma não aplicabilidade efetiva das técnicas comunicativas. Tal fato torna-se importante, já que reflete o perfil dos enfermeiros que repetem o paradigma da comunicação ineficaz. A enfermagem, ciência que tem por base o cui- 
dar, não se exime desse contexto, precisa da comunicação para se fundamentar como área autônoma principalmente para abolir o estereótipo atribuído pelo senso comum referente a profissão. Além disso, tem buscado se firmar como uma profissão racional e crítica e não meramente tecnicista, encontrando apoio no processo comunicativo que a dimensiona e a direciona ao cuidado holístico e humanitário quando o relacionamento interpessoal entre enfermeiros e pacientes hospitalizados é estabelecido.

Os estudos sobre comunicação precisam sair do âmbito acadêmico a fim de que seus resultados possam realmente ser utilizados para atingir a excelência do cuidado (Rossi, Batista, 2006; Stefanelli, 1993; Stefanelli, Carvalho, 2005), nesse caso a comunicação terapêutica.

Essa consiste na habilidade do profissional em usar seu conhecimento sobre comunicação, para ajudar a pessoa com tensão temporária, a conviver com outras pessoas e ajustar-se ao que não pode ser mudado e a superar os bloqueios e a auto-realização para enfrentar seus problemas (Stefanelli, 1993; Stefanelli, Carvalho, 2005). Porém muitas vezes os enfermeiros podem agir de maneira não comunicativa, requerendo atenção para que esse fato não ocorra e a comunicação atue como um instrumento de cuidar. Nesse estudo terá como objeto apenas a não-terapêutica, como forma de alertar os enfermeiros nas suas interações assistenciais.

Seguindo a teórica Stefanelli, as técnicas de comunicação terapêutica são classificadas em três grupos, a saber: Expressão, Clarificação e Validação. No primeiro grupo encontra-se as técnicas que ajudam a descrição da experiência e a expressão de pensamentos e sentimentos sobre essa. No grupo de Clarificação são apresentadas técnicas que ajudam o profissional a esclarecer o que for expresso pelo paciente. E no grupo de Validação são introduzidas as técnicas que permitem a existência de significação comum do que é expresso.

Assim como existem técnicas, estratégias ou guias para tornarem terapêutica a comunicação, há também, na comunicação diária, social e profissional, o que se pode chamar de comunicação nãoterapêutica. Alguns aspectos que caracterizam esse grupo existente em algumas atuações comunicativas. São eles: Não saber ouvir; Linguagem Inacessível; Dar conselhos; Julgar o comportamen- to; Manter-se na Defensiva; Pôr o Paciente à Prova; Mudar de Assunto Inadequadamente; Comunicação Unidirecional; Dupla Mensagem e Desconfirmação.

Assim o presente estudo busca ao caracterizar o processo comunicativo entre enfermeiros e pacientes no ambiente hospitalar, atentar os enfermeiros para sua conduta diante dos mais variados aspectos comunicativos no seu cotidiano. Estudos dessa natureza justificam-se pela busca da comunicação de fato terapêutica no âmbito assistencial. Assim objetivou-se: Caracterizar a comunicação não terapêutica entre enfermeiros e pacientes no ambiente hospitalar segundo os pressupostos de Stefanelli (1993).

\section{PROCEDIMENTOS METODOLOGICOS}

Estudo descritivo e exploratório, já que observa, conta, descreve e classifica os fenômenos estudados, bem como investiga a sua natureza complexa e os outros fatores com os quais se relacionam (Polit, Beck, Hungler, 2004). Portanto, a pesquisa descreve e investiga a comunicação entre enfermeiros e pacientes no ambiente hospitalar de forma a considerar os fatores internos e externos que interferem e/ou auxiliam a sua prática. Tem uma abordagem predominantemente quantitativa.

Foi realizado num hospital na região do Sertão Central estado do Ceará. A instituição foi escolhida inicialmente por ser campo de estágio da primeira autora e também por ser referência para a cidade a qual se localiza e demais regiões circunvizinhas. Na coleta de dados, foram selecionados os setores de clínica médica, clínica cirúrgica e obstetrícia, pois de acordo com o dimensionamento de pessoal da instituição, é direcionado um enfermeiro para os três setores e como o período de internação é de no mínimo dois dias, o contato com os enfermeiros torna-se mais fácil e conveniente e as possibilidades da utilização das técnicas comunicativas são maiores. A clínica cirúrgica possui 12 leitos e o setor obstétrico 16 leitos, sendo que oito estão na sala de pré-parto e parto e oito no alojamento conjunto. Já a clínica médica é composta por nove enfermarias com 32 leitos. O estudo foi desenvolvido no mês de Julho de 2008 nos turnos manhã, tarde e noite de acordo a escala de cada profissional observado. 
Optou-se pela técnica de observação sistemática não participante, que tem como característica básica o planejamento prévio e a utilização de anotações, recorrendo ao uso de recursos técnicos, mecânicos ou eletrônicos (Marconi, Lakatos, 2006). O observador deliberadamente se mantém na posição de observador e espectador, evitando se envolver ou deixar-se envolver com o objeto da observação. Para tanto, utilizou-se um check-list, adaptado de Stefanelli(1993) com as estratégias de comunicação terapêutica. Elaborou-se outro instrumento que contemplou os dados de identificação pessoal e profissional, além do procedimento observado.

Os dados foram agrupados em tabelas, as quais contemplaram os profissionais observados, as interações realizadas e os itens pertinentes à comunicação não terapêutica. As discussões além de caracterizarem o processo comunicativo dos enfermeiros ainda consideraram aspectos gerais, aplicados ao cotidiano da enfermagem no ambiente hospitalar.

Foram contempladas todas as normas da Resolução 196/96 do Conselho Nacional de Saúde do Ministério da Saúde do Brasil. Os enfermeiros assinaram os termos anteriormente ao inicio da coleta propriamente dita, porém não sabiam quando e nem qual procedimento exatamente estavam sendo avaliados. Além disso, é importante relatar que a pesquisadora adentrou no campo de pesquisa sete dias antes da coleta, para fazer parte da realidade observada. Esses cuidados foram tomados para que não houvesse mudanças drásticas por parte dos enfermeiros quanto à semiotécnica dos procedimentos por eles realizados. Os enfermeiros foram iden-

\begin{tabular}{|l|l|l|}
\hline ENFERMEIROS & \multicolumn{1}{|c|}{ INTERAÇŌES } & \multicolumn{1}{c|}{ TOTAL DE } \\
INTERACŌES
\end{tabular}
tificados com as letras A, B, C, D e E.

\section{RESULTADOS E DISCUSSÃO}

Participaram os enfermeiros dos setores de clínica médica, clínica cirúrgica e obstetrícia do hospital. A instituição conta com 14 enfermeiros; todos atuam nos setores especificados. De acordo com o período estipulado para a coleta, a pesquisadora teve contato com 08 profissionais. Destes, dois se recusaram em participar e um foi abordado no período noturno, depois de estar num plantão de 48 horas. Mediante rotina da instituição, os profissionais que estão nessas condições têm direito a descanso se não houver nenhuma intercorrência. Como o horário da coleta coincidiu com o repouso desse profissional, a pesquisa não pôde ser realizada com o mesmo.

Do total, cinco enfermeiros foram observados, destes, três eram do sexo feminino e dois do masculino; a faixa etária variou entre 24 e 56 anos e o período de exercício da profissão, oscilou de um a 22 anos. Desses dois trabalham integralmente na assistência hospitalar e três deles atuavam também na Estratégia Saúde da Família(ESF) da cidade. A seguir, a tabela 1 mostra as interações ocorridas entre enfermeiros e pacientes de acordo com cada profissional observado.

TABELA 01: Distribuição dos enfermeiros de acordo com as interações estabelecidas durante a assistência. Ceará, 2008
De acordo com a tabela 1 , foram observadas 21 interações entre enfermeiros e pacientes durante $o$ período de coleta. Destas, algumas se direcionam exclusivamente à prática comunicativa, como é o caso das orientações ao paciente sobre cirurgia, orientações às parturientes, às puérperas, à pacien- 
te que se submeteria à cesariana e também à visita aos pacientes.

Por outro lado, algumas das interações requereram além da comunicação, a semiotécnica, como foi o caso da sondagem vesical, da verificação da pressão arterial(PA) e temperatura, realização dos curativos, acompanhamento da evolução e trabalho de parto vaginal, punção venosa e vacinação das puérperas.

Como mostra a tabela, dos 05 profissionais, o que mais estabeleceu contato com os pacientes foi o enfermeiro $\mathrm{C}$, realizando nove das 21 interações totais. Dentre elas, citam-se: visita aos pacientes, orientações ao paciente sobre cirurgia, verificação da P.A e temperatura, toque vaginal, acompanhamento da evolução e do trabalho de parto normal, orientações à parturiente, orientações às puérperas e à paciente que se submeteria à cesariana e punção venosa.

$\mathrm{O}$ segundo profissional que mais promoveu interações com os pacientes foi o enfermeiro A, estabelecendo cinco delas, sendo citadas: visita aos pacientes, sondagem vesical, orientações ao paciente sobre cirurgia, verificação da P.A e avaliação das parturientes.

Os enfermeiros B e E realizaram três interações cada. O enfermeiro B executou: visita aos pacientes, realização de curativo cirúrgico I e realização de curativo cirúrgico II.

Já o Enfermeiro E, fez: vacinação das puérperas, sondagem vesical e realização de curativo contaminado. Por último o enfermeiro D estabeleceu uma interação que foi a punção venosa.

A seguir, a tabela 2 mostra os itens referentes à comunicação não-terapêutica, adaptados de Stefanelli, assim como, sua relação com as interações assistenciais estabelecidas entre pacientes e enfermeiros observados.
De acordo com a tabela foram encontradas 32 situações não terapêuticas. Observa-se que o enfermeiro A foi o profissional que mais itens nãoterapêuticos(11). O enfermeiro $\mathrm{E}$ apresentou sete situações, os profissionais $\mathrm{B}$ e $\mathrm{C}$, seis atuações e o enfermeiro D apenas dois, sendo o que menos vezes se enquadrou nos tópicos referentes à classificação não-terapêutica.

Usar Jargão Profissional foi o item mais praticado dentro da comunicação não-terapêutica, sendo visualizado por três vezes na prática dos enfermeiros A e D; por duas vezes pelos enfermeiros $\mathrm{B}$ e $\mathrm{C}$ e uma vez pelo enfermeiro $\mathrm{D}$. O segundo item que mais apareceu foi o item seis, Comunicação Unidirecional.

Já o item Oferecer Falsa Tranquilização, foi praticado duas vezes pelo enfermeiro A e uma vez pelos enfermeiros C e E. Mudar de Assunto Inadequadamente foi observado uma vez na prática assistencial dos enfermeiros A e E e duas vezes sendo praticado pelo profissional $\mathrm{B}$.

O item Dar Conselhos foi usado uma vez pelos enfermeiros A e C. Dar Resposta Estereotipada foi observado em uma situação nas interações do profissional A, quanto nas atuações do profissional E.

Não Saber Ouvir foi realizado apenas uma vez pelo enfermeiro B. O item Usar Termos Com Conotação De Julgamento foi usado uma única vez pelo enfermeiro B. Induzir Resposta foi praticado em uma situação pelo profissional A.

\section{DISCUSSÕES}

Foram observadas 21 interações comunicacionais durante a assistência de enfermagem em âmbito hospitalar. Mendoza, Castañón e Luis (2002), ratificam que é responsabilidade dos enfermeiros garantir o domínio sobre as condições e processos técnicos necessários para receber um
Tabela 2: Distribuição dos itens pertinentes à Comunicação Não-Terapêutica, mediante interações realizadas pelos enfermeiros.Ceará, 2008.

\begin{tabular}{|l|c|c|c|c|c|c|}
\hline \multicolumn{1}{|c|}{ GRUPOS COMUNICATIVOS } & \multicolumn{3}{c|}{ ENFERMEIROS } \\
\hline & A & B & C & D & E & Total \\
\hline Usar jargão profissional & 3 & 2 & 2 & 1 & 3 & 11 \\
\hline Comunicação unidirecional & 2 & - & 2 & 1 & 2 & 7 \\
\hline Oferecer falsa tranquuilização & 2 & - & 1 & - & 1 & 4 \\
\hline Mudar de assunto inadequadamente & 1 & 2 & - & - & 1 & 4 \\
\hline Dar conselhos & 1 & - & 1 & - & - & 2 \\
\hline Dar resposta estereotipada & 1 & - & - & - & 1 & 2 \\
\hline Não saber ouvir & - & 1 & - & - & - & 1 \\
\hline Usar termos com conotação de julgamento & - & 1 & - & - & - & 1 \\
\hline Induzir resposta & 1 & - & - & - & - & 1 \\
\hline TOTAL & $\mathbf{1 1}$ & $\mathbf{6}$ & $\mathbf{6}$ & $\mathbf{2}$ & $\mathbf{7}$ & $\mathbf{3 2}$ \\
\hline
\end{tabular}


serviço de saúde e estabelecer um processo de comunicação mais que informativo, favorável à interação que proporcione o compartilhar de expectativas, valores e sentimentos entre eles e os clientes. Neste sentido o processo comunicativo relacionado ao enfermeiro, é empregado em situações como: a entrevista, o exame físico, o planejamento da assistência, as orientações aos indivíduos, famílias e comunidades(Oliveira et al, 2005). Em suma ela perpassa todas as atividades de enfermagem.

Na tabela 2 referente aos grupos não-terapêuticos ocorreram um total de 32 vezes durante as interações observadas, independente de cada enfermeiro. Dentre eles dois chamaram bastante a atenção, visto que quando comparado com os demais ocorreram em número maior, a saber: Usar Jargão Profissional e Comunicação Unidirecional.

$\mathrm{O}$ item que mais apareceu independente do profissional que o executou foi o item Usar Jargão Profissional. É comum nas instituições de saúde o profissional utilizar linguagem inacessível, como jargões ou termos técnicos ou científicos quando interage com paciente (Stefanelli, Carvalho, 2005). A não compreensão desta forma especifica de comunicação parece tornar o paciente ansioso e, em alguns casos, pode causar sentimentos depressivos, ansiedade e até levá-lo ao isolamento (Macêdo, Pagliuca, 2005). Ao se remeter esse resultado para a interação com o paciente, deve-se considerar também que o uso predominante da linguagem técnica pode ser uma barreira na comunicação gerando medo, ansiedades, podendo refletir no prognóstico do paciente.

Apesar do hábito adquirido ainda na academia de utilizar termos técnicos ao se comunicarem, os enfermeiros precisam se policiar e discernirem como, quando e onde utilizá-los; no caso do contato com os pacientes estes não devem se fazer presentes já que se comportam como barreiras comunicativas.

O segundo item que também apareceu em um quantitativo importante foi o item Comunicação Unidirecional. A comunicação só poderá se efetuar eficazmente se essa perpassar de modo bilateral e horizontal na maior parte das interações.

Quando a comunicação unidirecional ocorre quando só o profissional falava, tem-se um monó-

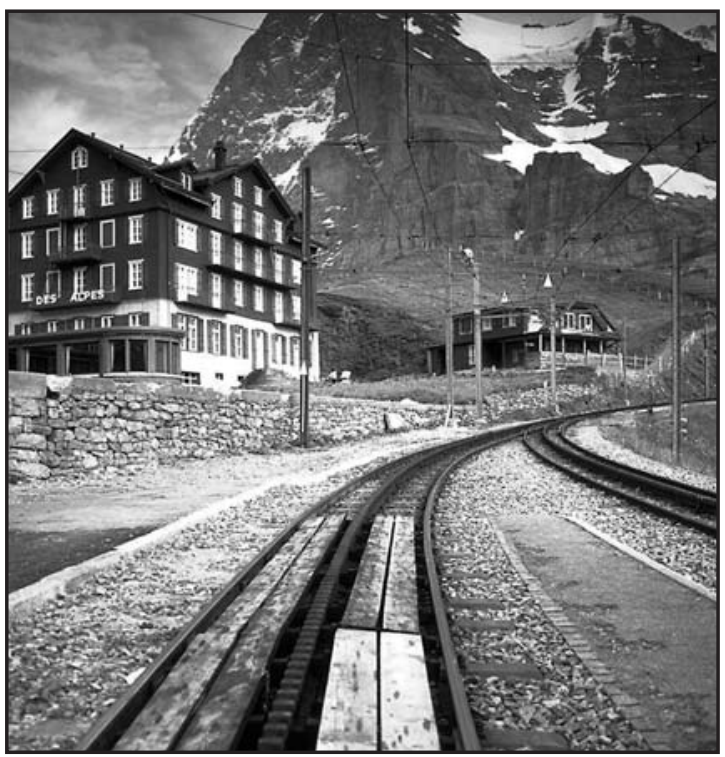

logo, uma vez que não é dada oportunidade ao paciente para se manifestar (Stefanelli, Carvalho, 2005). Este modo de comunicação torna-se mais fácil para o emissor, pois este detém o controle da situação. O paciente torna-se um elemento passivo quando recebe orientação sobre algum exame que irá fazer, mas não tem oportunidade de esclarecer suas dúvidas. O resultado em geral é sua não cooperação

A comunicação entre enfermeiros e pacientes, sobretudo no ambiente hospitalar, deve ser obrigatoriamente terapêutica, sendo esta, não um passatempo casual, mas sim uma atitude profissional deliberada e planejada, já que durante o processo de hospitalização, o paciente é afastado de seu ambiente familiar, das atividades sociais e profissionais, passando a vivenciar um novo cotidiano (Rossi, Batista, 2006).

Para que o enfermeiro possa corrigir sua deficiência na comunicação precisará, antes demais nada, ter consciência do fato e desenvolver-se na habilidade de ouvir reflexivamente.

O item Oferecer Falsa Tranquilização foi realizado pelo enfermeiro A, C e E. Para Stefanelli, essa ação do enfermeiro promove ou dificulta as respostas negativas do paciente (Stefanelli, 1993). Uma das características da enfermagem é lidar com diferentes questões emocionais não só do cliente como do próprio profissional. Para isso, 
deve lançar mão de instrumentos básicos da relação de ajuda que envolvem o processo comunicativo, como o diálogo para atingir a cura (Machado, Leitão, Holanda, 2005).

Mudar de Assunto Inadequadamente também foi observado. Mudar de assunto inadequadamente ou intercalar subitamente na conversa do paciente um assunto diferente, pode significar para este menosprezo de idéias, e não importância para com suas palavras. Em geral isso é feito inconscientemente ou não, para evitar um assunto que não se conhece ou não se domina (Stefanelli, Carvalho, 2005).

Mudar de assunto inadequadamente ou intercalar subitamente na conversa do paciente um assunto diferente, pode significar para este menosprezo de idéias, e não importância para com suas palavras. Em geral isso é feito inconscientemente ou não, para evitar um assunto que não se conhece ou não se domina.

Outro item que surgiu apenas uma vez foi Dar Conselhos. Dar conselhos ou dizer ao paciente o que ele deve fazer impede-o de tomar suas próprias decisões. Em geral, quando se emite conselhos a alguém, tem-se como base, julgamento e esquema de valores próprios (Stefanelli, Carvalho, 2005) .

A comunicação é importante para a pessoa hospitalizada tanto no que se refere à compreensão do jargão local quanto à segurança necessária ao paciente nessas condições. Assim, o profissional enfermeiro deve oferecer uma relação de ajuda, baseada no processo comunicativo, na qual, pelo menos uma das partes procura promover na outra o crescimento, o desenvolvimento, a maturidade, o melhor funcionamento e maior capacidade de enfrentar a vida (Paula, Furegato, Scatena, 2000).

O mesmo ocorre quando se dá uma opinião sobre a experiência ou questões que o paciente apresenta. O ideal é que se forneçam elementos ao paciente para que ele decida o que é mais adequado à situação por ele vivenciada.

Foi encontrado o item Dar Resposta Estereotipada, esta reação do enfermeiro, geralmente está relacionada, com seu estado emocional, e pode ser causada por problemas que nada têm a ver com o que o paciente fala (Stefanelli, Carvalho, 2005). Deve ser evitada, mas, quando ocorre, o enfermeiro poderá procurar corrigir-se dizendo ao paciente que está preocupado com algo, mas que tentará compreendê-lo (Bermúdez, Garcia, 2006).

Referente ao tópico Não Saber Ouvir,que apareceu apenas uma vez, o motivo do enfermeiro na maioria das vezes não saber ouvir o paciente pode significar que suas próprias necessidades são por ele colocadas acima das do paciente; nesse caso o paciente pode supor que o profissional não tem interesse por ele, e não se preocupa com suas necessidades (Rossi \& Batista, 2006; Macedo, Pagliuca, 2005).

O item Usar Termos com Conotação de Julgamento foi usado pelo enfermeiro B. Julgar o comportamento do paciente e emitir opinião própria sobre o mesmo tira-lhe o direito de tomar decisão.

Deve-se evitar na comunicação com o paciente o uso de termos que denotem julgamento. Termos como "bom" e "mau", "certo" e "errado" ou expressões verbais e não-verbais que indiquem aprovação ou reprovação são usados com base nos valores de quem os emite (Braga, Berti, Risso, Silva, 2009). A pessoa pode transmitir ao paciente a informação daquilo que ela gosta ou pensa e, o paciente, para agradá-la, pode passar a agir dessa maneira ocultando informações sobre si próprio e sobre seu estado de saúde.

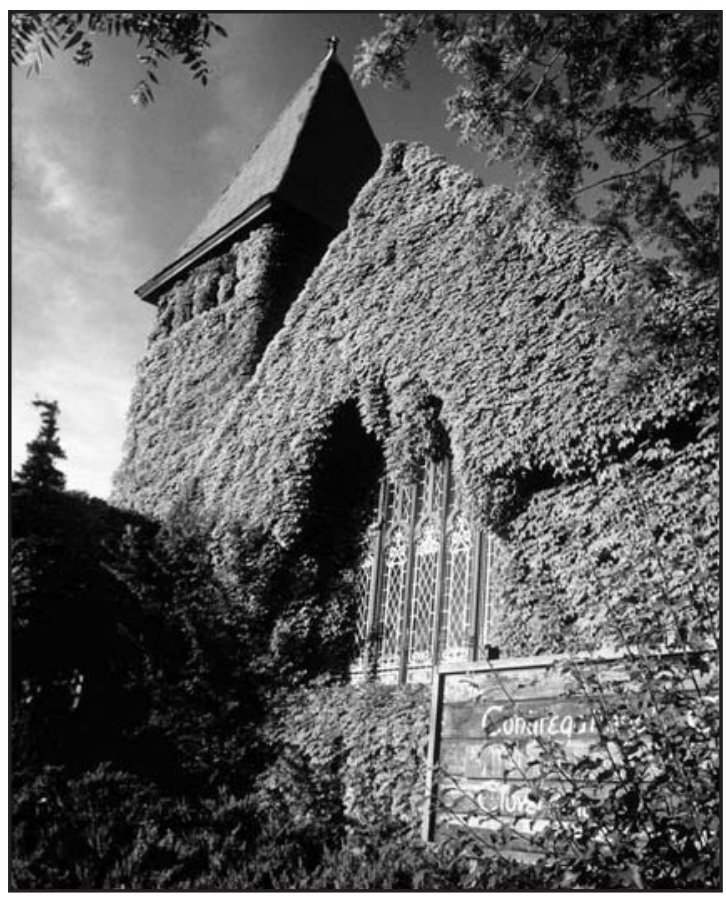


O item Induzir Resposta foi realizado pelo profissional A. Uma reflexão importante que deve ser dada é a sobrecarga pelo quais os profissionais da enfermagem estão submetidos. O que muitas vezes gera imediatismo nas respostas de interações comunicacionais.

Um estudo (Takaki, Sant'ana, 2004) mostrou que um dos fatores identificados como impossibilitador da empatia no cuidado ministrado pelos enfermeiros, foi a falta de tempo em função da grande demanda de clientes para serem atendidos.

\section{CONSIDERAÇÕES FINAIS}

De acordo com os dados apresentados e discutidos, constata-se que os itens não-terapêuticos estiveram presentes nas interações da maioria dos enfermeiros. Atenta-se ao fato de que, o mais utilizado foi o uso de jargão profissional. Constatou-se que, embora os enfermeiros não se atentem na maioria das vezes para as técnicas comunicativas até mesmo por possivelmente não conhecê-las, as utilizam cotidianamente de uma forma ou de outra. A comunicação é um instrumento básico para a enfermagem, e para que ela seja usada de maneira terapêutica os profissionais necessitam ter consciência das suas principais falhas.

\section{REFERÊNCIAS}

- Bermúdez ICN, Garcia GPR. (2006) La comunicación com los pacientes. Invest. Educ. Enferm., 24(1): 94-98.

- Braga EM, Berti HW, Risso ACMCR, Silva MJ (2009). Relações interpessoais da equipe de enfermagem em centro cirúrgico Rev. SOBECC,14(1):22-29.

- Chubaci RYS, Merighi MAB. (2002) A comunicação no pro- cesso da hospitalização do imigrante japonês. Rev. LatinoAm. de Enfermagem, 10(6):805-812.

- Macêdo KNF, Pagliuca LMF. (2005) Características da comunicação interpessoal entre profissionais de saúde e deficientes visuais. Revista Paulista de Enfermagem, 23(3): 221-226.

- Marconi MA, Lakatos EM. (2006) Metodologia do Trabalho Científico. Atlas, São Paulo (BR).

- Machado MMT, Leitão GCM, Holanda FUX (2005) O conceito de ação comunicativa: uma contribuição para a consulta de enfermagem. Rev. Latino-am. de enfermagem,13(5): 723728.

- Mendoza AZ, Castañón MAH, Luis MAV. (2002) Importância da comunicação na avaliação da qualidade da atenção de enfermagem e a satisfação da mulher depois do parto. In: $8^{\circ}$ Simpósio Brasileiro de Comunicação em Enfermagem. Anais...São Paulo.

- Oliveira PS et al. (2005) Comunicação terapêutica em enfermagem, revelada nos depoimentos de pacientes internados em centro de terapia intensiva. Rev. Eletrônica de Enfermagem, 7(1):54-63.

- Paula AAD, Furegato ARF, Scatena MCM (2008). Interação enfermeiro-familiar de paciente com comunicação prejudicada. Rev. Latino-Am. de Enfermagem, 8(4): 45-51.

- Polit DF, Beck CT, Hungler BP (2004). Fundamentos de pesquisa em enfermagem: métodos, avaliação e utilização. Artmed, Porto Alegre(BR).

- Rossi OS, Batista NA. (2006) O ensino da comunicação na graduação em medicina- uma abordagem. InterfaceComunicação, Saúde, Educação, 10(19):93-102.

- Sadala MLA, Stefanelli MC. (1996) Avaliação do ensino de relacionamento enfermeira-paciente. Rev. Latino-Am. de Enfermagem, 4(esp): 139-152.

- Stefanelli MC (1993). Comunicação com paciente - Teoria e ensino. Robe, São Paulo (BR).

- Stefanelli MG, Carvalho ECA. (2005) Comunicação nos Diferentes Contextos da Enfermagem. Manole, São Paulo(BR).

- Takaki MH, Sant'ana DM. (2004) A empatia como essência no cuidado prestado ao cliente pela equipe de enfermagem de uma unidade básica de saúde. Cogitare Enfermagem, 9(1): 7983.

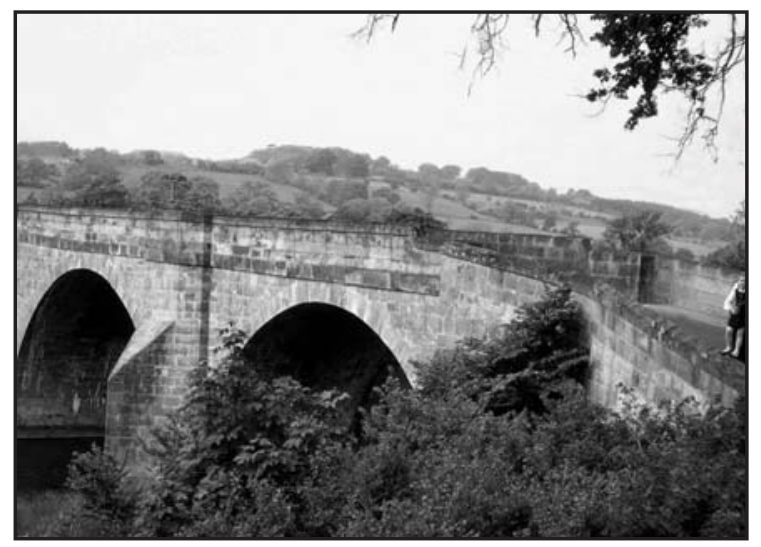

